TRANSACTIONS OF THE

AMERICAN MATHEMATICAL SOCIETY

Volume 350, Number 5, May 1998, Pages 1945-1956

S $0002-9947(98) 02019-4$

\title{
EXTENSION AND APPROXIMATION OF CR FUNCTIONS ON TUBE MANIFOLDS
}

\author{
ANDRÉ BOIVIN AND ROMAN DWILEWICZ
}

\begin{abstract}
A complete generalization of the classical Bochner theorem for infinite tubes is given.
\end{abstract}

\section{INTRODUCTION}

Let $\tau(N)=N \times i \mathbb{R}^{n} \subset \mathbb{C}^{n}, n \geq 2$, be the tube over an embedded submanifold $N$ of $\mathbb{R}^{n}$. Let us denote by $\operatorname{ch}(S)$ the convex hull of a set $S$. By $\operatorname{Int} \operatorname{ch}(S)$ we will mean the interior taken in the smallest dimensional space which contains $\operatorname{ch}(S)$, and we define (following [Ka]) the almost convex hull of $S$ by $\operatorname{ach}(S):=S \cup \operatorname{Int} \operatorname{ch}(S)$. The main theorem of this paper can now be stated as follows:

Main Theorem. Let $N$ be a connected submanifold of $\mathbb{R}^{n}$ of class $C^{2}$. Then any continuous $C R$ function on the tube $\tau(N)$ can be continuously extended to a $C R$ function on $\tau(\operatorname{ach}(N))$. As a consequence of this, any $C R$ function on $\tau(N)$ can be almost uniformly approximated by holomorphic polynomials.

Our main theorem gives a complete generalization of the classical Bochner tube theorem ([Bo], $[\mathrm{BM}],[\mathrm{H}$, Theorem 2.5.10]) which states that any holomorphic function on a tube $\Omega+i \mathbb{R}^{n} \subset \mathbb{C}^{n}$, with $\Omega$ a domain in $\mathbb{R}^{n}$, extends holomorphically to the tube over the convex hull of $\Omega$. Note that also in our theorem, whenever the interior in $\mathbb{R}^{n}$ of $\operatorname{ch}(N)$ is nonempty, the extension obtained must then be holomorphic. It should also be mentioned that Kazlow $[\mathrm{Ka}]$ has obtained a generalization of Bochner's theorem but under somewhat more restrictive and complicated hypotheses, and that Baouendi and Trèves $[\mathrm{BT}]$ have proved a microlocal version of Bochner's theorem.

The main theorem can also be considered as a version of the edge-of-the-wedge theorem. It is well-known that tubular manifolds, the classical Bochner tube theorem and the classical edge-of-the-wedge theorem of Bogolyubov [Bogo] play important roles, and extend far beyond complex analysis. For instance, N.N. Bogolyubov, a mathematical physicist, proved his theorem to solve some problems in physics in connection with quantum field theory and dispersion relations.

As a consequence of our version of Bochner's theorem, we will get the property that CR functions on tubular manifolds can be approximated almost uniformly by holomorphic polynomials. This property does not hold in general for arbitrary

Received by the editors February 6, 1996 and, in revised form, August 7, 1996.

1991 Mathematics Subject Classification. Primary 32C16; Secondary 32D10, 32D15.

Key words and phrases. Tubular manifolds, CR functions.

Research partially supported by NSERC grants. 
CR manifolds; some additional assumptions are needed. See, for instance, [DG]. Note that the main theorem of [DG] already implies some approximation results for tubular manifolds (see [BD]).

In the proof of the main theorem, we will consider several cases with respect to the geometry of the base manifold $N(\S 5)$. Our proof makes use of the lemma of the folding screen, in the sense of Kazlow [Ka], but with weaker assumptions on the class of CR functions (§3); it also requires a characterization of minimal tube manifolds (in the sense of Tumanov) (see $\S 4$ ); it then proceeds with an induction argument on the codimension of the original manifold $(\S 5)$. We will also use some results from CR theory, such as the edge-of-the-wedge theorem of Airapetjan-Henkin and the extension theorem of Tumanov.

Acknowledgement. The authors would like to thank Professor Jürgen Leiterer for stimulating discussions which led to this paper, and Professor C. Denson Hill for useful conversations, especially about the class of CR functions in our main theorem.

\section{Definitions AND NOtATion}

CR manifolds. We will assume in this paper that all manifolds are of class $C^{2}$. An embedded submanifold $M$ of $\mathbb{C}^{n}$ is called Cauchy-Riemann (CR) if the complex dimension of the complex part of the tangent fibre $T_{p} M$ to $M$ at $p$ does not depend on $p$, i.e.,

$$
\operatorname{dim}_{\mathbb{C}}\left(T_{p} M \cap \sqrt{-1} T_{p} M\right)=l(p) \equiv l=\text { const. }
$$

The constant $l$ is called the CR dimension of $M$ and is denoted by $\operatorname{dim}_{\mathrm{CR}} M$. A submanifold $\widetilde{M} \subset M$ is called a $C R$ submanifold of $M$ if $\widetilde{M}$ is a CR submanifold of $\mathbb{C}^{n}$. A CR submanifold $M$ of $\mathbb{C}^{n}$ is called a generic $C R$ submanifold if $\operatorname{codim}_{\mathbb{R}} M=$ $n-\operatorname{dim}_{\mathrm{CR}} M$.

Following $[\mathrm{Tu}]$, we say that a $\mathrm{CR}$ manifold $M$ is minimal at $p$ if there are no CR submanifolds $\widetilde{M}$ passing through $p$ with $\operatorname{dim}_{\mathrm{CR}} \widetilde{M}=\operatorname{dim}_{\mathrm{CR}} M$ but $\operatorname{dim}_{\mathbb{R}} \widetilde{M}<$ $\operatorname{dim}_{\mathbb{R}} M$.

By a $C R$ function $f: V \longrightarrow \mathbb{C}, V$ open in $M$, we mean a continuous function which satisfies the tangential Cauchy-Riemann equations on $M$; i.e., $\int_{V} f \wedge \bar{\partial} \beta=0$ for any smooth and compactly supported form $\beta$ on $V$. If $f$ is of class $C^{1}$ on $M$, then it satisfies the standard tangential Cauchy-Riemann equations. For more details about $\mathrm{CR}$ manifolds and CR functions, see [Bogg].

Wedges. We assume that $M$ is a generic submanifold contained in an open connected subset $U$ of $\mathbb{C}^{n}$, with $\operatorname{dim}_{\mathrm{CR}} M=n-k$ and $\operatorname{codim}_{\mathbb{R}} M=k$. Let the coordinates in $\mathbb{C}^{n}$ be $z=x+i y=\left(z_{1}, \ldots, z_{n}\right)=\left(x_{1}+i y_{1}, \ldots, x_{n}+i y_{n}\right)$. Suppose that the local defining equations for $M$ are

$$
M:=\left\{z \in U ; \quad \rho_{1}(z)=\ldots=\rho_{k}(z)=0\right\},
$$

where the real-valued functions $\left\{\rho_{j}\right\}$ satisfy $\rho_{j} \in C^{2}(U)$ for $j=1, \ldots, k$ and $\bar{\partial} \rho_{1} \wedge \ldots \wedge \bar{\partial} \rho_{k} \neq 0$ everywhere in $U$.

Let $C$ be an open convex cone in $\mathbb{R}^{k}$. By the wedge with edge $M$ we mean the domain

$$
\mathcal{W}(U, C)=\left\{z \in U ; \quad \rho(z)=\left(\rho_{1}(z), \ldots, \rho_{k}(z)\right) \in C\right\}
$$


Sometimes it is convenient to describe wedges by using the defining functions for $M$ as in (2.1). Namely,

$$
\mathcal{W}=\left\{z \in U ; \quad \rho_{j}(z)>0 \quad \text { for } j=1, \ldots, k\right\} .
$$

We note that $M$ is the intersection of "ribs": i. e. $M=\bigcap_{j} \bar{M}_{j}$, where

$$
M_{j}=\left\{z \in U ; \quad \rho_{j}(z)>0, \rho_{\alpha}(z)=0, \alpha \neq j\right\}, \quad j=1, \ldots, k .
$$

\section{EDGE-OF-THE-WEDGE THEOREM AND THE LEMMA OF THE FOLDING SCREEN}

In this section we prove the lemma of the folding screen. Several versions of this lemma can be found in the literature. See, for instance, [H, Lemma 2.5.11] and [Ka, Lemma 4.1, Proposition 5.1]; see also [Ko] and [BT, Remark 3.1]. Our version of the lemma is closest to that of Kazlow. In Kazlow's version, it is assumed that the CR functions are $C^{\infty}$, and his proof (as far as we know) cannot be carried out for continuous CR functions.

Our proof of the lemma will be based on the edge-of-the-wedge theorem of Airapetjan-Henkin [AH1], which we now formulate using the notation and definitions from $\S 2$.

Edge-of-the-Wedge Theorem [AH1, Th. 1]. For any $\varepsilon>0$ there exists a neighborhood $U_{\varepsilon}$ of the manifold $M$ so that any continuous function $f$ on the compact set $\bigcup_{1}^{k} \bar{M}_{j}$ which is a $C R$ function on the manifold $\bigcup_{1}^{k} M_{j}$ extends continuously on $\mathbb{C}^{n}$ to a function $F$ which is holomorphic in the domain

$$
\left\{z \in U_{\varepsilon} ; \quad \rho_{j}(z)>\varepsilon \sum_{\alpha \neq j} \rho_{\alpha}(z), \quad j=1,2, \ldots, k\right\} .
$$

Remark 3.1. Just after Theorem 1 in [AH1], we can read the following comments: "In the case when all the functions $\left\{\rho_{j}\right\}$ are pluriharmonic, i.e., when $\partial \bar{\partial} \rho_{j}=0$ for $1 \leq j \leq k$, the conclusion of the edge-of-the-wedge theorem holds even when $\varepsilon=0$ and follows from the classical theorem of S.N. Bernstein on separate analyticity (see [AR], where, however, it is also assumed that $f$ is sufficiently smooth)." In $[\mathrm{AH} 2$, Theorem $7 \mathrm{~b}]$, an integral formula for the holomorphic extension is given in this special case if in addition $M$ is assumed to be totally real.

In the rest of the section we use the following notation. Let $\left\{A_{j}\right\}_{j=0}^{k}$ be a set of points in $\mathbb{R}^{n}$. We denote by $l_{i j}$ the open segment joining $A_{i}$ and $A_{j}$, by $\bar{l}_{i j}$ the closed segment, and $\hat{l}_{i j}=A_{i} \cup l_{i j}$.

Lemma 3.2 (lemma of the folding screen). Let $\left\{A_{j}\right\}_{j=0}^{k}(k \leq n)$ be a convex linearly independent set of points in $\mathbb{R}^{n}(n \geq 2)$. Then any continuous function on $\tau\left(\bigcup_{j} \bar{l}_{0 j}\right)$ which is $C R$ on $\tau\left(\bigcup_{j} l_{0 j}\right)$ can be continuously extended to $\tau\left(\operatorname{ch}\left(\bigcup_{j} \hat{l}_{0 j}\right) \cup\right.$ $\left.\bigcup_{j} A_{j}\right)$ in such a way that it is $C R$ on $\tau\left(\operatorname{ch}\left(\bigcup_{j} l_{0 j}\right)\right)$.

Remark 3.3. The continuity of functions on $\tau\left(\operatorname{ch}\left(\bigcup_{j} \hat{l}_{0 j}\right) \cup \bigcup_{j} A_{j}\right)$ should be understood to mean that the functions are continuous on the tube over any compact simplex $\Delta \subset \operatorname{ch}\left(\bigcup_{j} \hat{l}_{0 j}\right) \cup \bigcup_{j} A_{j}$; i.e. we assume only non-tangential limits at boundary points.

Proof of Lemma 3.2. Without loss of generality we can assume that $A_{0}=(0, \ldots, 0)$ and $A_{j}=(0, \ldots, 0,1,0, \ldots, 0)$, where the " 1 " is in the $j^{\text {th }}$ position. Define the 
manifolds

$$
M:=\left\{z \in \mathbb{C}^{n} ; \quad x_{1}=0, \ldots, x_{k}=0\right\},
$$

and

$$
M_{j}:=\left\{z \in \mathbb{C}^{n} ; \quad 0<x_{j}<1, x_{\alpha}=0, \alpha \neq j\right\}, \quad j=1, \ldots, k .
$$

Using the edge-of-the-wedge theorem, the fact that the functions defining $M$ are obviously pluriharmonic (see Remark 3.1), and the property that the geometry of the tube is "the same on each level", there exists a number $0<a \leq 1$ so that any continuous function $f$ on the set $\bigcup_{1}^{k} \bar{M}_{j}$ which is CR on the manifold $\bigcup_{1}^{k} M_{j}$ extends continuously on $\mathbb{C}^{n}$ to a function $F$ which is holomorphic in the domain

$$
\left\{z \in \mathbb{C}^{n} ; \quad x_{j}>0, x_{1}+\ldots+x_{k}<a, \quad j=1, \ldots, k\right\} .
$$

The main step in the proof of the lemma is to show that it holds when $n=k=2$. So assuming $n=k=2$, let

$$
T_{\max }=\left\{\left(z_{1}, z_{2}\right) \in \mathbb{C}^{2} ; \quad x_{1}>0, x_{2}>0, x_{1}+x_{2}<a_{\max }\right\}, \quad 0<a_{\max } \leq 1,
$$

be the maximal tube to which all continuous functions defined on $\tau\left(\bar{l}_{01} \cup \bar{l}_{02}\right)$ and CR on $\tau\left(l_{01} \cup l_{02}\right)$ extend. Note that by symmetry with respect to $x_{1}$ and $x_{2}$, we can assume that the tube is indeed of the form given above. We want to prove that $a_{\max }=1$. Suppose that $a_{\max }<1$ and let $\mathcal{W}$ be any wedge whose boundary is of the form

$$
\begin{aligned}
& \partial \mathcal{W}=\tau\left(\left\{a_{\max } \leq x_{1} \leq 1, x_{2}=0\right\}\right) \cup \tau\left(\left\{\left(1 / a_{\max }\right) x_{1}+b x_{2}=1,0 \leq x_{1} \leq a_{\max }\right\}\right), \\
& \text { where } b>1 / a_{\max } \text {. }
\end{aligned}
$$

The CR functions restricted to $\partial \mathcal{W}$ satisfy the assumptions of the edge-of-thewedge theorem; in particular, they are continuous at each point $p_{0}$ of the edge $\tau\left(\left\{\left(a_{\max }, 0\right)\right\}\right)$. The continuity follows, for example, from the fact that there exists an analytic disc whose boundary lies on $M$ and which passes through $p_{0}$. The real and imaginary parts of the extension must then be solutions of the classical Dirichlet problem with continuous boundary conditions. The continuity at the edge follows. Therefore, using the edge-of-the-wedge theorem of Airapetjan-Henkin, CR functions defined on $\partial \mathcal{W}$ can be holomorphically extended to the inside of the wedge in some tubular neighborhood of the edge $\tau\left(\left\{\left(a_{\max }, 0\right)\right\}\right)$. By symmetry, we have the same property at points of $\tau\left(\left\{\left(0, a_{\max }\right)\right\}\right)$. Applying the classical Bochner tube theorem, we get a contradiction with the maximality of $a_{\max }$.

To complete the proof of the lemma, we reduce the remaining cases to the case just proved, i.e., $n=k=2$.

If $k=2$ and $n \geq 2$, we can consider the CR functions on our tube as CR (or holomorphic) functions on the corresponding tubes in $\mathbb{C}^{2}$ with some dependence on the variables $\left(i y_{3}, \ldots, i y_{n}\right)$, which will be taken as parameters. It is easy to see that the dependence of the extended CR function (the extension obtained from the case $n=k=2$ ) on the parameters is of the same class as that of the original CR function.

The remaining cases when $k \leq n$ are obtained immediately from the preceding case by a slicing argument. The details are left to the reader.

The next two lemmas are consequences of the lemma of the folding screen. Their hypotheses reflect situations that we will encounter during the proof of our main 
theorem in $\S 5$-A. If $X$ is a topological space, we denote by $\operatorname{Int}_{X}$ the interior relative to $X$.

Lemma 3.4. Let $l \leq n$ and let $N$ be a submanifold of $\mathbb{R}^{l} \subset \mathbb{R}^{n}$. Assume that $\operatorname{Int}_{\mathbb{R}^{l}}(\operatorname{ch} N) \neq \emptyset$ and that $U$ is an open convex domain in $\mathbb{R}^{n}$ such that $\operatorname{Int}_{N}(\bar{U} \cap N)$ $\neq \emptyset$. Assume that $f$ is a continuous function on

$$
[\operatorname{ach}(N) \cup U] \times i \mathbb{R}^{n}=\left[\operatorname{Int}_{\mathbb{R}^{l}}(\operatorname{ch}(N)) \cup N \cup U\right] \times i \mathbb{R}^{n}
$$

which is CR on each "term" of the above decomposition. Then there exists a continuous extension of $f$ to $[\operatorname{ach}(\operatorname{ach}(N) \cup U)] \times i \mathbb{R}^{n}$ which is holomorphic in

$$
\left[\operatorname{Int}_{\mathbb{R}^{n}}(\operatorname{ch}(\operatorname{ach}(N) \cup U))\right] \times i \mathbb{R}^{n} .
$$

Remark 3.5. Continuity has to be understood in the sense of existence of nontangential limits at boundary points as described in Remark 3.3. Given a tube $\tau(T)=T \times i \mathbb{R}^{n}$ and a continuous CR function $f$ on $\tau(T)$, a continuous (in the above sense) extension of $f$ to $\tau(\operatorname{ach}(T))$ which is continuous and CR in $\tau(\operatorname{Int}(\operatorname{ch}(T)))$ will be called a continuous $C R$ extension of $f$. In particular, if $\operatorname{Int}_{\mathbb{R}^{n}} \operatorname{ch}(T) \neq \emptyset$, then the extension will be called a continuous holomorphic extension.

Proof. From the hypothesis, it follows that we can choose $n+1$ linearly independent points $\left\{A_{j}\right\}_{j=0}^{n}$ such that $A_{0} \in \operatorname{Int}_{N}(N \cap \bar{U}) ; A_{1}, \ldots, A_{l} \in \operatorname{ch}(N) ; A_{l+1}, \ldots, A_{n} \in$ $U$. Note that $f$ restricted to $\tau\left(\bigcup_{j} l_{0 j}\right)$ is CR, and consequently, by the lemma of the folding screen, $f$ can be continuously extended to a function $F$ on $E=$ $\tau\left(\operatorname{ch}\left(\bigcup_{j} \hat{l}_{0 j}\right) \cup \bigcup_{j} A_{j}\right)$ such that $F$ is holomorphic on $E_{0}=\tau\left(\operatorname{ch}\left(\bigcup_{j} l_{0 j}\right)\right)$. We claim that the extension of $f$ obtained from any other set of linearly independent points $B_{0}, \ldots, B_{n}$ chosen as above must agree with $F$. Indeed, let $\lambda_{0 j}$ denote the open segment joining $B_{0}$ and $B_{j}$, let $\widetilde{E}=\tau\left(\operatorname{ch}\left(\bigcup_{j} \hat{\lambda}_{0 j}\right) \cup \bigcup_{j} B_{j}\right)$, let $\widetilde{F}$ be the CR extension of $\left.f\right|_{\tau\left(\cup \bar{\lambda}_{0 j}\right)}$ to $\widetilde{E}$, and let $P$ be a common point of $E$ and $\widetilde{E}$. Without loss of generality we can assume that $P \in E \cap \widetilde{E} \cap \mathbb{R}^{n}$. Now we apply the lemma of the folding screen twice, first to the set $S_{1}$ formed by the two segments $\operatorname{ch}\left(\left\{A_{n}, P\right\}\right)$ and $\operatorname{ch}\left(\left\{A_{n}, B_{n}\right\}\right)$, and next to the set $S_{2}$ formed by the two segments $\operatorname{ch}\left(\left\{B_{n}, P\right\}\right)$ and $\operatorname{ch}\left(\left\{B_{n}, A_{n}\right\}\right)$. The $\mathrm{CR}$ function on $\tau\left(\operatorname{ch}\left(\left\{A_{n}, B_{n}\right\}\right)\right)$ is taken to be simply the restriction of $f$, the $\mathrm{CR}$ function on $\tau\left(\operatorname{ch}\left(\left\{A_{n}, P\right\}\right)\right)$ will be the restriction of $F$, and finally the CR function on $\tau\left(\operatorname{ch}\left(\left\{B_{n}, P\right\}\right)\right)$ will be the restriction of $\widetilde{F}$. We then obtain two $\mathrm{CR}$ functions on $\tau\left(\operatorname{ch}\left(\left\{A_{n}, B_{n}, P\right\}\right)\right)$. They must agree on $\tau\left(\operatorname{ch}\left(\left\{A_{n}, B_{n}\right\}\right)\right)$, and consequently they must be the same. This implies that $F$ and $\widetilde{F}$ must agree at $P$.

To complete the proof of the lemma, it suffices to note that the union over all the simplexes formed by all combinations of $n+1$ linearly independent points chosen as above contains $[\operatorname{ach}(\operatorname{ch}(N) \cup N \cup U)] \times i \mathbb{R}^{n}$.

Lemma 3.6. Let $L$ be a real subspace of $\mathbb{R}^{n}$ with $\operatorname{dim}_{\mathbb{R}} L<n$ and such that $\mathbb{R}^{l}$ and $L$ span $\mathbb{R}^{n}$, where $l<n$ and $\operatorname{dim}_{\mathbb{R}}\left(\mathbb{R}^{l} \cap L\right)>0$. Let $N$ be a submanifold of $\mathbb{R}^{n}$ which does not lie globally in a smaller dimensional subspace and with the property that $N=\left(N \cap \mathbb{R}^{l}\right) \cup(N \cap L)$. Moreover assume that $\operatorname{Int}_{\mathbb{R}^{l}}\left(\operatorname{ch}\left(N \cap \mathbb{R}^{l}\right)\right) \neq \emptyset$ and $\operatorname{Int}_{L}(\operatorname{ch}(N \cap L)) \neq \emptyset$. Then any continuous function defined on the tube

$$
\left[\operatorname{Int}_{\mathbb{R}^{l}} \operatorname{ch}\left(N \cap \mathbb{R}^{l}\right) \cup \operatorname{Int}_{L} \operatorname{ch}(N \cap L) \cup N\right] \times i \mathbb{R}^{n}
$$


which is CR on each "term" of the above decomposition can be continuouslyholomorphically extended to the tube

$$
\operatorname{Int}_{\mathbb{R}^{n}}\left\{\operatorname{ch}\left[\operatorname{ch}\left(N \cap \mathbb{R}^{l}\right) \cup \operatorname{ch}(N \cap L) \cup N\right]\right\} \times i \mathbb{R}^{n} .
$$

Proof. Choose $n+1$ linearly independent points $\left\{A_{k}\right\}_{k=0}^{n}$ such that $A_{0} \in \mathbb{R}^{l} \cap L \cap N$; $A_{1}, \ldots, A_{l} \in \operatorname{ch}\left(N \cap \mathbb{R}^{l}\right) \cup\left(N \cap \mathbb{R}^{l}\right) ; A_{l+1}, \ldots, A_{n} \in \operatorname{ch}(N \cap L) \cup(N \cap L)$. Following the proof of Lemma 3.4, we apply the lemma of the folding screen to the simplex spanned by $A_{0}, \ldots, A_{n}$. Following again the argument in the proof of Lemma 3.4 , we see that any two such extensions obtained from any two sets of points chosen as above must agree. We complete the proof by taking the union of all such simplexes.

\section{Minimal tube manifolds}

In this section we prove the following proposition.

Proposition 4.1. Let $N$ be a submanifold of $\mathbb{R}^{n}$ and $\tau(N)$ be the tube over $N$. If there exists a CR submanifold $M$ of $\tau(N)$ such that $\operatorname{dim}_{C R} M=\operatorname{dim}_{C R} \tau(N)$, then for each $p_{0} \in M$ there is a neighborhood $U$ of $\pi\left(p_{0}\right)$ in $N$ which is contained in an affine space $L$ with $\operatorname{dim}_{\mathbb{R}} L=\operatorname{dim}_{\mathbb{R}} M-\operatorname{dim}_{C R} M$, where $\pi: \mathbb{C}^{n} \longrightarrow \mathbb{R}^{n}, \pi(z)=\Re z$, is the natural projection. Conversely, if $N$ is locally around $\pi\left(p_{0}\right)$ contained in an affine space $L$, then there is a CR submanifold $M$ of $\tau(N)$ through $p_{0}$ as above.

As an immediate consequence of the proposition, we have the following:

Corollary 4.2. Let $N$ be a submanifold of $\mathbb{R}^{n}$ and $\tau(N)$ be the tube over $N$. Then $\tau(N)$ is not minimal at $p_{0} \in \tau(N)$ if and only if there is a neighborhood of $\pi\left(p_{0}\right)$ in $N$ that is contained in an affine space $L \subset \mathbb{R}^{n}$, where $\operatorname{dim}_{\mathbb{R}} L<n$.

Remark 4.3. Note that in the proposition, the manifold $M$ which has the smallest real dimension is uniquely determined. This follows from the paper of Sussmann $[\mathrm{Su}]$.

Example 4.4. If a tube manifold $\tau(N)$ is not minimal at each point, then it does not necessarily follow that $N$ is globally contained in an affine space $N \subset \mathbb{R}^{n}$ with $\operatorname{dim}_{\mathbb{R}} L<n$. It is very easy to construct a curve $N$ in $\mathbb{R}^{3}$ such that locally $N$ is lying in a plane but globally not. The tube over such $N$ is not minimal at each point.

Proof of the proposition. Necessity: Let $N$ be a submanifold of $\mathbb{R}^{n}$ with $\operatorname{codim}_{\mathbb{R}} N$ $=k$. Without loss of generality we can assume that $p_{0}=0$ and $N$ (and also $\tau(N)$ ) is locally given by a system of equations

$$
x_{\alpha}=\varphi_{\alpha}\left(x_{k+1}, \ldots, x_{n}\right) \quad \text { for } \quad \alpha=1, \ldots, k,
$$

where

$$
\varphi_{1}(0)=\ldots=\varphi_{k}(0)=0, \quad d \varphi_{1}(0)=\ldots=d \varphi_{k}(0)=0 .
$$

Assume, as in the hypothesis of the proposition, that there exists a CR submanifold $M$ of $\tau(N)$ with $n-k=\operatorname{dim}_{\mathrm{CR}} \tau(N)=\operatorname{dim}_{\mathrm{CR}} M$ but $2(n-k) \leq m=$ $\operatorname{dim}_{\mathbb{R}} M \leq \operatorname{dim}_{\mathbb{R}} \tau(N)=2 n-k$. This implies that

$$
\{0\} \times \mathbb{C}^{n-k} \subset T_{0} M \subset i \mathbb{R}^{k} \times \mathbb{C}^{n-k} \subset \mathbb{C}^{n},
$$


and $T_{0} M$ can be described by the following system of linear equations:

$$
\begin{cases}x_{\alpha}=0 & \text { for } \quad \alpha=1, \ldots, k \\ b_{\beta, 1} y_{1}+\ldots+b_{\beta, k} y_{k}=0 & \text { for } \quad \beta=1, \ldots, 2 n-k-m,\end{cases}
$$

where the coefficients $b_{\beta \gamma}$ are real and the matrix $\left(b_{\beta \gamma}\right)$ has rank $2 n-k-m$. Without any loss of generality we can assume that the second part of (4.3) is of the form

$$
y_{\beta}=c_{\beta, 2 n-k-m+1} y_{2 n-k-m+1}+\ldots+c_{\beta, k} y_{k} \quad \text { for } \quad \beta=1, \ldots, 2 n-k-m .
$$

Since $M$ is a submanifold of $\tau(N)$ and because of (4.4), $M$ can be given by a system of equations

$$
\begin{cases}x_{\alpha}=\varphi_{\alpha}\left(x_{k+1}, \ldots, x_{n}\right) & \text { for } \alpha=1, \ldots, k \\ y_{\beta}=\psi_{\beta}\left(x_{k+1}, \ldots, x_{n}, y_{2 n-k-m+1}, \ldots, y_{n}\right) & \text { for } \beta=1, \ldots, 2 n-k-m .\end{cases}
$$

By our assumption $\operatorname{dim}_{C R} M=n-k$ and by (4.5) we can find the form of $(0,1)$ vector fields $\bar{X}$ tangent to $M$. Namely we have $\bar{X}=a_{1} \partial / \partial \bar{z}_{1}+\ldots+a_{n} \partial / \partial \bar{z}_{n}$, where

$$
\begin{aligned}
a_{\alpha}= & \frac{\partial \varphi_{\alpha}}{\partial x_{k+1}} a_{k+1}+\ldots+\frac{\partial \varphi_{\alpha}}{\partial x_{n}} a_{n} \quad \text { for } \quad \alpha=1, \ldots, k \\
a_{\beta}= & \frac{\partial \psi_{\beta}}{\partial y_{2 n-k-m+1}} a_{2 n-k-m+1}+\ldots+\frac{\partial \psi_{\beta}}{\partial y_{k}} a_{k}+ \\
& +\left(\frac{\partial \psi_{\beta}}{\partial y_{k+1}}-i \frac{\partial \psi_{\beta}}{\partial x_{k+1}}\right) a_{k+1}+\ldots+\left(\frac{\partial \psi_{\beta}}{\partial y_{n}}-i \frac{\partial \psi_{\beta}}{\partial x_{n}}\right) a_{n} \\
& \text { for } \beta=1, \ldots, 2 n-k-m,
\end{aligned}
$$

where the derivatives are evaluated at $p \in M$ which corresponds to the parameters $\left(x_{k+1}, \ldots, x_{n}, y_{2 n-k-m+1}, \ldots, y_{n}\right)$. Since $\operatorname{dim}_{\mathrm{CR}} M=n-k$, the above system should give exactly $k$ independent restrictions on $a_{1}, \ldots, a_{n}$. The first $k$ equations give exactly these restrictions, and this implies that the other equations should be identically satisfied for all $a_{k+1}, \ldots, a_{n}$. If we take $a_{1}, \ldots, a_{k}$, evaluated from the first $k$ equations, into the second set of equations, regroup the terms with respect to $a_{k+1}, \ldots, a_{n}$, equate the corresponding coefficients to zero, and finally, equate the real and imaginary parts to zero, we obtain, respectively, the following systems of differential equations:

$$
\begin{aligned}
& \frac{\partial \varphi_{\beta}}{\partial x_{\gamma}}=\frac{\partial \psi_{\beta}}{\partial y_{\gamma}}+\frac{\partial \varphi_{2 n-k-m+1}}{\partial x_{\gamma}} \frac{\partial \psi_{\beta}}{\partial y_{2 n-k-m+1}}+\ldots+\frac{\partial \varphi_{k}}{\partial x_{\gamma}} \frac{\partial \psi_{\beta}}{\partial y_{k}} \\
& \text { for } \beta=1, \ldots, 2 n-k-m \quad \text { and } \gamma=k+1, \ldots, n,
\end{aligned}
$$

and

$$
\frac{\partial \psi_{\beta}}{\partial x_{\gamma}}=0 \quad \text { for } \quad \beta=1, \ldots, 2 n-k-m \quad \text { and } \quad \gamma=k+1, \ldots, n .
$$

The system (4.7) gives that the functions $\psi$ depend on $y$ only, i.e.,

$$
\psi_{\beta}=\psi_{\beta}\left(y_{2 n-k-m+1}, \ldots, y_{n}\right) .
$$


Next, using the second part of (4.2), the system (4.6) yields

$$
\frac{\partial \psi_{\beta}}{\partial y_{\gamma}}=0 \quad \text { for } \quad \beta=1, \ldots, 2 n-k-m \quad \text { and } \quad \gamma=k+1, \ldots, n .
$$

Finally, we integrate the equations from (4.6) with respect to suitable $x$ 's, namely, the $\gamma^{\text {th }}$ equations with respect to $x_{\gamma}$, and using the first part of (4.2) we obtain

$$
\varphi_{\beta}=\varphi_{2 n-k-m+1} \frac{\partial \psi_{\beta}}{\partial y_{2 n-k-m+1}}+\ldots+\varphi_{k} \frac{\partial \psi_{\beta}}{\partial y_{k}} \quad \text { for } \quad \beta=1, \ldots, 2 n-k-m .
$$

We note that the functions $\varphi$ depend on $x$ only and the functions $\psi$ on $y$ only. Take $y=0$, and put $c_{\beta, \gamma}=\frac{\partial \psi_{\beta}(0)}{\partial y_{\gamma}}$. Then we get

$$
\varphi_{\beta}=c_{\beta, 2 n-k-m+1} \varphi_{2 n-k-m+1}+\ldots+c_{\beta, k} \varphi_{k} \quad \text { for } \quad \beta=1, \ldots, 2 n-k-m,
$$

which gives that $N$ near $p$ lies in a linear subspace of $\mathbb{R}^{n}$. The dimension of this subspace is $n-(2 n-k-m)=m-(n-k)=\operatorname{dim}_{\mathbb{R}} M-\operatorname{dim}_{\mathrm{CR}} \tau(N)$.

Sufficiency: If the manifold $N$, locally around $\pi\left(p_{0}\right)$, is contained in an affine subspace $L \subset \mathbb{R}^{n}$ of dimension $l<n$, then without loss of generality we can assume that $L=\mathbb{R}^{l}$. The tube near $\pi\left(p_{0}\right)$ is of the form

$$
\tau(N)=\left\{\left(x_{1}+i y_{1}, \ldots, x_{l}+i y_{l}, i y_{l+1}, \ldots, i y_{n}\right) ; \quad\left(x_{1}, \ldots, x_{l}, 0, \ldots, 0\right) \in N\right\} .
$$

Obviously, for $p_{0}=\left\{\left(x_{1}^{0}+i y_{1}^{0}, \ldots, x_{l}^{0}+i y_{l}^{0}, i y_{l+1}^{0}, \ldots, i y_{n}^{0}\right)\right.$, we can construct a CR submanifold $M$ of $\tau(N)$,

$$
M=\left\{\left(x_{1}+i y_{1}, \ldots, x_{l}+i y_{l}, i y_{l+1}^{0}, \ldots, i y_{n}^{0}\right) ; \quad\left(x_{1}, \ldots, x_{l}, 0, \ldots, 0\right) \in N\right\},
$$

which is of the same CR dimension as $\tau(N)$ but of smaller real dimension. Consequently $\tau(N)$ is not minimal at $p_{0}$.

The proposition is proved.

\section{5-A. Proof of the main theorem-EXtension}

0. General preparation. Let $N$ be a submanifold of $\mathbb{R}^{n}$ of real codimension $k$, $0 \leq k=\operatorname{codim}_{\mathbb{R}} N \leq n$, and let $\tau(N)$ be the tube over $N$.

We shall prove the theorem by induction with respect to $k$. Obviously the theorem is true if $k=0$, since in this case $N$ is an open domain in $\mathbb{R}^{n}$ and the theorem is exactly the classical Bochner tube theorem (see $\S 1$ ). Our induction hypothesis is that the theorem is true for manifolds $\widetilde{N}$ in $\mathbb{R}^{s}$ when $\operatorname{dim}_{\mathbb{R}} \mathbb{R}^{s}-$ $\operatorname{dim}_{\mathbb{R}} \tilde{N}=s-\operatorname{dim}_{\mathbb{R}} \widetilde{N}<k$. Our goal is to prove the theorem for manifolds $N$ in $\mathbb{R}^{n}$ such that $n-\operatorname{dim}_{\mathbb{R}} N=k$.

We will proceed by distiguishing two cases:

1. There is a minimal point on $\tau(N)$. Let $p_{0} \in \tau(N)$ be a point at which $\tau(N)$ is minimal. Obviously we can assume that $p_{0} \in N$. By the theorem of Tumanov $[\mathrm{Tu}]$, any continuous $\mathrm{CR}$ function defined on $\tau(N)$ near $p_{0}$ can be holomorphically extended to an open wedge in $\mathbb{C}^{n}$ with edge $\tau(N)$ locally near $p_{0}$. Denote the interior of the extension set by $U$. Since the extension set, constructed in [Tu], is constructed using analytic discs attached to $M$, it is clear that $U$ can be assumed to be a tube over an open set in $\mathbb{R}^{n}$, i.e., $U=\tau(\pi(U))$. We note that $\overline{U \cap \mathbb{R}^{n}}=\overline{\pi(U) \cap \mathbb{R}^{n}}$ contains the point $p_{0}$. (Recall that $\pi: \mathbb{C}^{n} \longrightarrow \mathbb{R}^{n}$ is the natural projection). 
Now we take the maximal open and connected extension set $U_{\max }$ containing $U$ that satisfies the following conditions: (i) at each point $p \in \operatorname{Int}\left(\tau(N) \cap \overline{U_{\max }}\right)$, there exists a closed convex cone $C_{p} \subset \mathbb{R}^{k}$ (with non-empty interior) and a small neighbourhood $V$ of $p$ in $\tau(N) \cap \overline{U_{\max }}$ such that if $C_{q}$ is the cone with vertex $q \in V$ obtained by a rigid translation of $C_{p}$, then $C_{q} \backslash\{q\}$ near $p$ should be contained in $U_{\max }$; and (ii) any continuous CR function on $\tau(N)$ can be continuously and holomorphically extended to $U_{\max }$. In other words, $U_{\max }$ contains some wedge with edge $\operatorname{Int}\left(\tau(N) \cap \overline{U_{\max }}\right)$. The "opening" of the wedge could get smaller and smaller as the point approaches the boundary of the edge in $\tau(N)$. We note that $U_{\max }$ is nonempty because it contains $U$. We can assume that $U_{\max }$ is convex, since if not, we apply Bochner's tube theorem. We can also assume that $U_{\max }$ is a tube, i.e., $U_{\max }=\tau\left(\pi\left(U_{\max }\right)\right)$.

Remark. A monodromy problem can appear with such definition of $U_{\max }$. This can be easily avoided by restricting $U_{\max }$ to the tube over a tubular neighborhood of $N$ in $\mathbb{R}^{n}$ (tubular neighborhood in the sense of differential geometry), and slightly modifying the property of convexity of $U_{\max }$. In order not to obscure our proof, we leave $U_{\max }$ as it was originally defined.

Let $S=N \cap \overline{U_{\max }}$. Note that this set is closed and has nonempty interior in $N$. If $S=N$, then the conclusion of the theorem holds since $\operatorname{Int}(\tau(\operatorname{ch}(N))) \subset U_{\max }$.

Consequently, it is enough to consider the case $S \neq N$. This implies that $\overline{\operatorname{Int}_{N} S} \neq N$, and since $N$ is connected, $\overline{\operatorname{Int}_{N} S} \cap\left(N \backslash \operatorname{Int}_{N} S\right)$ is nonempty.

1a. Suppose there is a minimal point $q_{0}$ in $\overline{\operatorname{Int}_{N} S} \cap\left(N \backslash \operatorname{Int}_{N} S\right)$. If $N$ is a hypersurface, then we use the result of $[\operatorname{Tr}]$ and the uniqueness of extension of CR functions to extend CR functions defined on $\tau(N)$ to at least one side of the hypersurface near $q_{0}$. The extension can be to the same side as $U_{\max }$ or not; in either case, we get a contradiction with the maximality of $U_{\max }$.

If $N$ is of higher codimension, then using the result of [Tu], we obtain that there is an open wedge $\mathcal{W}$ with edge $\tau(N)$ near $q_{0}$ such that any CR function defined on $\tau(N)$ can be holomorphically extended to $\mathcal{W}$. Put $Q=N \cap \overline{\mathcal{W}}$. We have $q_{0} \in \operatorname{Int}_{N} Q$ and consequently there is a point $q_{1} \in \operatorname{Int}_{N} Q \cap \operatorname{Int}_{N} S$. In a neigborhood of $q_{1}$ in $N$ we have two wedges with edge $\tau(N)$ : one is $\mathcal{W}$ and the second one comes from $U_{\max }$. Then using the-edge-of-the-wedge theorem in the version of [AH1] (see $\S 3$ ) or in the version of Epstein (see $[\mathrm{E}],[\mathrm{R}]$ ), we obtain that any CR function on $\tau(N)$ holomorphically extends to an open convex wedge $\widetilde{\mathcal{W}}$ which intersects both the wedges: $\widetilde{\mathcal{W}} \cap \mathcal{W} \neq \emptyset$ and $\widetilde{\mathcal{W}} \cap U_{\max }$ near $q_{1}$. Again we obtain a contradiction with the maximality of $U_{\max }$.

1b. Now assume there are no minimal points in $\overline{\operatorname{Int}_{N} S} \cap\left(N \backslash \operatorname{Int}_{N} S\right)$. Fix any point $q_{0} \in \overline{\operatorname{Int}_{N} S} \cap\left(N \backslash \operatorname{Int}_{N} S\right)$, by Corollary 4.2, there is a neighborhood $N_{0}$ of $q_{0}$ in $N$ that is contained in an affine space of $\mathbb{R}^{n}$ of real dimension $<n$. Let $l$ be the smallest dimension of the space which contains any neigborhood of $q_{0}$ and assume that $N_{0}$ is contained in this space (we decrease $N_{0}$ if necessary). Without loss of generality we can assume that this space is $\mathbb{R}^{l} \subset \mathbb{R}^{n}$. We have that $\operatorname{Int}_{\mathbb{R}^{l}}\left(\operatorname{ch}\left(N_{0}\right)\right) \neq 0$. We shall identify $N_{0} \subset \mathbb{R}^{l}$ with $N_{0} \times\{(0, \ldots, 0)\} \subset \mathbb{R}^{n}$ when it does not lead to any confusion.

The tube over $N_{0}$ is of the form $N_{0} \times i \mathbb{R}^{n} \subset \mathbb{C}^{n}$ and is contained in $\mathbb{R}^{l} \times\{0\} \times$ $i \mathbb{R}^{l} \times i \mathbb{R}^{n-l} \simeq \mathbb{C}^{l} \times i \mathbb{R}^{n-l} \subset \mathbb{C}^{n}$. Let $\tau^{\prime}\left(N_{0}\right)$ be the tube over $N_{0}$ in $\mathbb{C}^{l}$, i.e., $\tau^{\prime}\left(N_{0}\right)=$ 
$N_{0} \times i \mathbb{R}^{l}$, and let $\tau_{y^{\prime}}^{\prime}\left(N_{0}\right)=\tau^{\prime}\left(N_{0}\right) \times\left\{i y^{\prime}\right\} \subset \mathbb{C}^{n}$, where $y^{\prime}=\left(y_{l+1}, \ldots, y_{n}\right)$. Any CR function on $\tau(N)$ generates a $\mathrm{CR}$ function on $\tau_{y^{\prime}}^{\prime}\left(N_{0}\right)$ for $y^{\prime} \in \mathbb{R}^{n-l}$. We can apply the induction assumption to $\tau_{y^{\prime}}^{\prime}\left(N_{0}\right)$, so we get that any continuous CR function on $\tau_{y^{\prime}}^{\prime}\left(N_{0}\right)$ extends continuously and holomorphically to $\tau_{y^{\prime}}^{\prime}\left(\operatorname{ach}\left(N_{0}\right)\right) \subset \mathbb{C}^{l} \times\left\{i y^{\prime}\right\}$ and is continuous with respect to $y^{\prime}$. The continuity with respect to $y^{\prime}$ follows easily from methods of extension of CR functions from minimal manifolds (see [Tu], [DH]). Thus, near $q_{0}$ in $\tau(N)$, one holomorphic extension of CR functions comes from the definition of $U_{\max }$, and the second CR extension to $\operatorname{Int}_{\mathbb{R}^{l}}\left(\operatorname{ch}\left(N_{0}\right)\right) \times i \mathbb{R}^{n}$ comes from the above description.

The hypotheses of Lemma 3.4 are thus all satisfied, and consequently we obtain that $\bar{U}_{\max } \cap N$ contains a neighborhood of $q_{0}$ in $N$. This contradicts the maximality of $U_{\max }$ at $q_{0}$.

This completes the proof of the theorem when there exists a minimal point on $\tau(N)$.

2. There are no minimal points on $\tau(N)$. From Corollary 4.2 , for each point $p \in N$ there is an affine subspace $L_{p}$ passing through $p \in N$ with $\operatorname{dim}_{\mathbb{R}} L_{p}<n$ and $N \subset L_{p}$ near $p$. Obviously this does not mean that $N$ is entirely contained in a single affine space of $\mathbb{R}^{n}$ of dimension $<n$ (see Example 4.4). Let $L$ be an affine space such that $N \subset L$ with the smallest possible dimension. If $\operatorname{dim}_{\mathbb{R}} L<n$, the theorem holds by the induction assumption and continuous dependence on parameters. So it is enough to prove the theorem in the case when $\operatorname{dim}_{\mathbb{R}} L=n$.

Fix any point $p_{0} \in N$ and let $V_{\max }, p_{0} \in V_{\max }$, be the maximal open connected subset of $N$ which is contained in an $(n-1)$-dimensional affine subspace of $\mathbb{R}^{n}$. We denote by $l$ the smallest dimension of the affine space which contains $V_{\max }$, so we have $\operatorname{Int}\left(\operatorname{ch}\left(V_{\max }\right)\right) \neq \emptyset$ in this affine space. Without loss of generality we can assume that the affine space containing $V_{\max }$ is $\mathbb{R}^{l}$. We note that $l$ is not necessarily $n-1$, but obviously we have $0 \leq l \leq n-1$. By the induction assumption, for each fixed $\left(y_{l+1}, \ldots, y_{n}\right) \in \mathbb{R}^{n-l}$, any CR function on $\tau(N)$ can be holomorphically extended to the tube $\operatorname{ch}\left(V_{\max }\right) \times i \mathbb{R}^{l} \times\left\{\left(i y_{l+1}, \ldots, i y_{n}\right)\right\} \subset \mathbb{C}^{l} \times\left\{\left(i y_{l+1}, \ldots, i y_{n}\right)\right\} \subset$ $\mathbb{C}^{n}$. Take $q_{0} \in \overline{V_{\max }} \cap\left(N \backslash V_{\max }\right)$. Such a point exists since $N$ does not lie entirely in $\mathbb{R}^{n-1}$. Since all points on $N$ are not minimal (this is the assumption in this case), there exist a neighborhood $N_{q_{0}}$ of $q_{0}$ in $N$ and an affine space $L_{q_{0}}$ such that $N_{q_{0}}$ is contained in $L_{q_{0}}$, with $\operatorname{dim}_{\mathbb{R}} L_{q_{0}}<n$, and such that $N_{q_{0}}$ is not contained in any affine space of smaller dimension (this follows from Corollary 4.2). Consequently $\operatorname{Int}\left(\operatorname{ch}\left(N_{q_{0}}\right)\right)$ is an open subset of $L_{q_{0}} \subset \mathbb{R}^{n}$ and, by our induction hypothesis, any CR function on $\tau(N)$ extends near $q_{0}$ to a CR function in the tube $\operatorname{ach}\left(N_{q_{0}}\right) \times i \mathbb{R}^{n}$.

We note that $\operatorname{dim}_{\mathbb{R}}\left(\mathbb{R}^{l} \cap L_{q_{0}}\right)>0$, since $V_{\max } \cap N_{q_{0}} \neq \emptyset$ and $N_{q_{0}} \subset L_{q_{0}}$. Consequently, all assumptions of Lemma 3.6 are satisfied. After application of this lemma we obtain that there is a tube over an open subset $U \subset \mathbb{R}^{n}$ to which CR functions from our assumptions holomorphically extend. Moreover $U$ satisfies the following condition: $\bar{U} \cap N$ contains a neigborhood of $q_{0}$ in $N$. Using this fact, this case can be reduced to a situation similar to the one found in the case 1(b).

This completes the proof of the extension part of the main theorem.

\section{5-B. Proof of the main theorem-APproximation}

When $\operatorname{Int}(\operatorname{ch}(N)) \neq \emptyset$ in $\mathbb{R}^{n}$, the approximation theorem follows immediately from the extension part of the theorem and the fact that $\operatorname{Int}(\operatorname{ch}(N))$ is geometrically convex. 
To prove the theorem when $\operatorname{Int}(\operatorname{ch}(N))=\emptyset$ in $\mathbb{R}^{n}$, let $L \subset \mathbb{R}^{n}$ be the smallest dimensional space that contains $\operatorname{ch}(N)$. So we have that $\operatorname{Int}(\operatorname{ch}(N)) \neq \emptyset$ in $L$. Without loss of generality we can assume that $L=\mathbb{R}^{l}$. From above, for each fixed $\left(i y_{l+1}, \ldots, i y_{n}\right)$, any continuous $\mathrm{CR}$ function $f$ on $\tau(N)=N \times i \mathbb{R}^{n}$ can be approximated by functions $F\left(z^{\prime}, i y^{\prime \prime}\right)=F\left(z_{1}, \ldots, z_{l}, i y_{l+1}, \ldots, i y_{n}\right)$ which are holomorphic polynomials with respect to $z_{1}, \ldots, z_{l}$. The functions $F\left(z^{\prime}, i y^{\prime \prime}\right)$ can be chosen in such a way that the dependence on $y^{\prime \prime}$ is continuous (by using, for instance, a Mittag-Leffler star type approximation [M, vol. III, Ch. 8, sec. 41]) and moreover, if $y^{\prime \prime} \in \mathbb{R}^{l}$ with $\left|y^{\prime \prime}\right| \leq r$ for some $r>0$, then the function $F\left(z^{\prime}, i y^{\prime \prime}\right)$ is a holomorphic polynomial in $z^{\prime}$ with continuous coefficients (the degree of this polynomial depends on $r$ ). Now applying the Weierstrass theorem to each coefficient, we get that the function $F\left(z^{\prime}, i y^{\prime \prime}\right)$ can be almost uniformly approximated by holomorphic polynomials in all variables $z=\left(z_{1}, \ldots, z_{n}\right)$, and consequently the CR function $f$ also can be approximated by such polynomials. This completes the proof of the approximation part of the theorem.

\section{REFERENCES}

[AH1] R.A. Airapetjan and G.M. Henkin, Analytic continuation of CR-functions through the "edge of the wedge", Soviet Math. Dokl. 24 (1981), 128 - 132. MR 82k:32039

[AH2] R.A. Airapetjan and G.M. Henkin, Integral representations of differential forms on Cauchy-Riemann manifolds and the theory of CR-functions, Russian Math. Surveys 39 (1984), no. 3, 41 - 118. MR 86b:32003

[AR] N.I. Ahiezer and L.I. Ronkin, Separately analytic functions of several variables, and "edge of the wedge" theorems, Russian Math. Surveys 28 (1973), no. 3, 27 - 44. MR 54:7847

[BT] M. S. Baouendi and F. Trèves, A microlocal version of Bochner's tube theorem, Indiana Univ. Math. J. 31 (1982), 885 - 895. MR 84b:35025

[Bo] S. Bochner, A theorem on analytic continuation of functions in several variables, Ann.of Math. 39 (1938), $14-19$.

[BM] S. Bochner and W.T. Martin, Several Complex Variables, Princeton University Press, Princeton, N.J. (1948). MR 10:366a

[Bogg] A. Boggess, CR Manifolds and the Tangential Cauchy-Riemann Equations, CRC Press, Studies in Advanced Mathematics (1991). MR 94e:32035

[Bogo] N. N. Bogolyubov, Introduction to the Theory of Quantized Fields, GITTL, Moscow (1957), English translation, Interscience, New York, 1959. MR 20:5047; MR 22:1349

[BD] A. Boivin and R. Dwilewicz, Holomorphic approximation of CR functions on tubular submanifolds of $\mathbb{C}^{2}$, Annales Polonici Math. 55 (1991), 11 - 18. MR 93b:32017

[DG] R. Dwilewicz and P.M. Gauthier, Global holomorphic approximations of CR functions on CR manifolds, Complex Variables 4 (1985), 377 - 391. MR 88b:32041

[DH] R. Dwilewicz and C. D. Hill, The normal type function for CR manifolds, preprint.

[E] H. Epstein, Generalization of the "edge-of-the-wedge" theorem, J. Mathematical Phys. 1 (1960), $524-531$. MR 22:10626

[H] L. Hörmander, An Introduction to Complex Analysis in Several Variables (Third Ed.), North-Holland Mathematical Library 7 (1989). MR 91a:32001

[Ka] M. Kazlow, CR functions and tube manifolds, Trans. Amer. Math. Soc. 255 (1979), 153 - 171. MR 80m:32001

[Ko] H. Komatsu, A local version of Bochner's tube theorem, J. Fac. Sci. Univ. Tokyo Sect. 1A Math. 19 (1972), 201 - 214. MR 47:5297

[M] A.I. Markushevich, Theory of functions of a complex variable, Chelsea Publishing Company, New York, N.Y. (1985). MR 56:3258 (earlier printing)

[R] W. Rudin, Lectures on the edge-of-the-wedge theorem, CBMS - AMS, No. 6, (1971). MR 46:9389

[Su] H.J. Sussmann, Orbits of families of vector fields and integrability of distributions, Trans. Amer. Math. Soc. 180 (1973), 171 - 188. MR 47:9666 
[Tr] J.M. Trépreau, Sur le prolongement holomorphe des fonctions $C$ - $R$ définies sur une hypersurface réelle de classe $C^{2}$ dans $\mathbb{C}^{n}$, Invent. Math. 83 (1986), 583 - 592. MR 87f:32035

$[\mathrm{Tu}]$ A. E. Tumanov, Extension of $C R$ functions into a wedge from a manifold of finite type, Math. USSR Sbornik 64 (1989), 129 - 140. MR 89m:32037

Department of Mathematics, University of Western Ontario, London, Ontario, N6A 5B7, CANADA

E-mail address: boivin@uwo.ca

Institute of Mathematics, Polish Academy of Sciences, Śniadeckich 8, P.O. Box 137, 00-950 WARsaW, Poland

E-mail address: rd@impan.gov.pl 